\title{
New Frontiers at the Interface of General Relativity and Quantum Optics
}

\author{
C. Feiler • M. Buser · E. Kajari · W.P. Schleich • \\ E.M. Rasel · R.F. O'Connell
}

Received: 27 August 2009 / Accepted: 19 November 2009 / Published online: 6 January 2010

(C) Springer Science+Business Media B.V. 2009

\begin{abstract}
In the present paper we follow three major themes: (i) concepts of rotation in general relativity, (ii) effects induced by these generalized rotations, and (iii) their measurement using interferometry. Our journey takes us from the Foucault pendulum via the Sagnac interferometer to manifestations of gravito-magnetism in double binary pulsars and in Gödel's Universe. Throughout our article we emphasize the emerging role of matter wave interferometry based on cold atoms or Bose-Einstein condensates leading to superior inertial sensors. In particular, we advertise recent activities directed towards the operation of a coherent matter wave interferometer in an extended free fall.
\end{abstract}

Keywords General relativity · Sagnac interferometer - Atom interferometer - Sagnac time delay · Gödel Universe · Gravito-magnetism

\section{Introduction}

When Faust is about to sign his pact with the devil, he is asked to use his own blood as ink, see Goethe (1808). The devil explains:

Blut ist ein ganz besondrer Saft.

Blood is a quite peculiar juice.

In the same vein time has always played and still continues to play a rather peculiar role in physics. The concept of an absolute time prevalent in the mechanics of Newton ultimately

C. Feiler $(\bowtie) \cdot$ M. Buser · E. Kajari · W.P. Schleich

Institut für Quantenphysik, Universität Ulm, Albert-Einstein-Allee 11, 89069 Ulm, Germany

e-mail: cornelia.feiler@uni-ulm.de

E.M. Rasel

Institut für Quantenoptik, Leibniz Universität Hannover, Welfengarten 1, 30167 Hannover, Germany

R.F. O'Connell

Department of Physics and Astronomy, Louisiana State University, Baton Rouge, LA 70803-4001, USA 
had to give way to the distinction between coordinate and proper time-a trademark of special as well as general relativity. The fact that time depends on the observer had already been anticipated by William Shakespeare who writes in Act 3 of As you like it, Bartlett and Kaplan (2002):

Time travels in diverse paces with diverse persons.

I'll tell you who Time ambles withal, who time trots withal,

Who Time gallops withal, and who he stands still withal.

Nowhere clearer do we see the difference between the past, the present and the future than in the light cone description of special relativity. Nevertheless, the problem of the arrow of time has not found a convincing solution yet. As Edward FitzGerald points out in his poem The Rubiáyát of Omar Khayyám:

The Moving Finger writes; and, having writ,

Moves on: Nor all your Piety nor Wit

Shall lure it back to cancel half a Line

Nor all your Tears wash out a Word of it.

On first sight one might think that general relativity supports this notion of an arrow of time and that it rules out the possibility of time travel; however, in 1949 Kurt Gödel showed that using an exact solution of Einstein's field equations "[...] it is theoretically possible [...] to travel into the past". This unusual feature is closely linked to the concept of rotation in general relativity. In the present paper we address various such concepts starting from the rotation of a coordinate system. We then turn to gravitational fields induced by a rotating mass distribution; finally we address the Gödel solution where the whole universe rotates. We also analyze methods of measuring the effects of the rotation using light and matter interferometers.

Our paper is organized as follows: In Sect. 2 we highlight mechanical and optical rotation sensors by focusing on the Foucault pendulum and the Sagnac interferometer. We then briefly discuss the state of the art of atom interferometers with Bose-Einstein condensates (BEC) and advertise a novel experimental apparatus designed by the QUANTUS Group for creating a BEC in free fall. In this way the concept of a freely falling elevator which was so important in the development of general relativity has been promoted from a "Gedanken" experiment into a real experiment. We dedicate Sect. 3 to a brief discussion of the effects of gravito-magnetism such as the Lense-Thirring effect starting from Einstein's field equations in the weak-field limit. Here, we only allude to the recent tests of the Lense-Thirring effect using Gravity Probe B and LAGEOS and LAGEOS II since this work is already covered by other articles in the book. Instead, we focus on the observation of gravito-magnetic effects in two-body systems such as double binaries. We then turn to the discussion of Gödel's Universe and illustrate by help of light cone diagrams how to perform time travel. Moreover, we visualize light propagation in this unusual metric. Needless to say we do not live in a Gödel Universe; nevertheless, it would be still interesting to create a laboratory system to study the propagation of light in this unusual and time travel allowing spacetime metric. Such an idea might become reality due to an analogy between Maxwell's equations in a dielectric medium and Maxwell's equations in a metric field. We conclude in Sect. 5 by summarizing our main results and give one more historical perspective on the central role of the weak equivalence principle.

Needless to say in a review such as the present one, i.e. space limitations make it impossible for us to present detailed derivations. As a consequence we can only but refer to the literature for more information. 
On a lighter note it is amusing but not too surprising that in a paper dedicated to the discussion of rotation a great variety of spirals make their appearance in unexpected places. For example, atom interferometry can be described most conveniently with the help of the Feynman path integral. An essential ingredient of this formulation is the Cornu spiral. Moreover, in the Gravity Probe B experiment geared towards the measurement of the Lense-Thirring effect the loxodrome appears. Finally the null geodesics in Gödel's Universe are helices giving rise to unusual views of objects.

\section{Rotation Sensors}

In the present section, we provide a historic overview over rotation sensors. We start by discussing the Foucault pendulum, briefly mention the original Sagnac effect and close with a concise introduction into the modern field of atom interferometers and BECs.

\subsection{Foucault Pendulum}

In 1851 Jean Bernard Léon Foucault (1819-1868) demonstrated to a stunned public the rotation of the Earth using a pendulum hanging from the ceiling of the Pantheon, see Foucault $(1851,1878)$. However, his experiment in the Pantheon was only the repetition of one performed already on January 6, 1851 at 2 a.m. in the basement of his mother's house at the corner of rue de Vaugirard and rue d'Assas in Paris. Foucault noted the event in his diary, see Aczel (2003):

Two o'clock in the morning, the pendulum has moved in the direction of the diurnal motion of the heavenly sphere.

Foucault had quite a remarkable career, despite the fact that he had no formal scientific training in natural sciences. He had studied medicine, but since he could not see blood turned to theoretical medicine. He made his living as an assistant to Alfred Donné who was a professor of medicine specializing in microscopy. One of the many problems they were facing was to illuminate plants underneath a microscope in order to take photographs. At that time, this task was a non-trivial endeavor, since photography had just been developed independently by Henry Fox Talbot in England and by Louis-Jacques-Mandé Daguerre in France. Foucault solved the illumination problem by developing a carbon-arc electric lamp which later on was even used in the Paris opera for a sun rise in Giacomo Meyerbeer's "Le Prophète".

Another problem of the Daguerre method of photography was the fact that it was impossible to take pictures of people due to exposure times up to half an hour. Together with Armand Hippolyte Louis Fizeau, Foucault found a method to reduce the exposure time to twenty seconds. In this way it becomes possible for the first time to take pictures of human beings.

Obviously the year 1851 was an extremely successful one for Foucault. Not only did he achieve instant fame with his pendulum experiment but he was also awarded a permanent research position which he held till his death. Indeed, he was appointed by the emperor Napoléon III as Physicist Attached to the Imperial Observatory in Paris. Moreover, in the same year Foucault also invented the mechanical gyroscope. Hence, he can be considered as an important contributor to the Gravity Probe B satellite launched in 2004 and discussed in Sect. 3. 


\subsection{Sagnac Interferometer}

In 1913 Georges Sagnac performed an experiment based on a light interferometer to measure the effect of rotation on the arrival times of two counter-propagating light rays. A sketch of his apparatus is shown in Fig. 1. In this device, light emitted by a mercury-arc lamp (O) is split by a prism (J) into two counter-propagating light beams. After successive reflections at the mirrors $(\mathrm{M})$, the beams are recombined by the same prism $(\mathrm{J})$ such that interference fringes on the photographic plate $\left(\mathrm{PP}^{\prime}\right)$ emerge.

Sagnac found that when he started to rotate the table with a constant rotation rate, these fringes moved. His discovery is a consequence of the fact that the light which propagates against the rotation returns to the starting point before the light that has to catch up with the rotation.

The fact stands out most clearly for the case of a circular light path of length $P \equiv 2 \pi r$. In the absence of rotation the light with velocity $c$ takes the time

$$
T=\frac{P}{c}=\frac{2 \pi r}{c}
$$

to complete the round. When the table rotates with the rate $\Omega$ the paths of the two light beams get extended or shortened by an amount $\delta P=\Omega T r=\Omega T \cdot c T /(2 \pi)$ leading to the path length difference

$$
2 \cdot \delta P=\frac{c}{\pi} \Omega T^{2}
$$

experienced by the two counter-propagating beams.

With the wave vector $k \equiv \omega / c$ we find from (2) for the phase shift $\Delta \varphi_{\mathrm{L}} \equiv k \cdot 2 \cdot \delta P$ the expression

$$
\Delta \varphi_{\mathrm{L}}=\frac{1}{\pi} \omega \Omega T^{2}
$$

Fig. 1 Sketch of the original light interferometer Sagnac presented in his second paper, see Sagnac (1913a, 1913b)

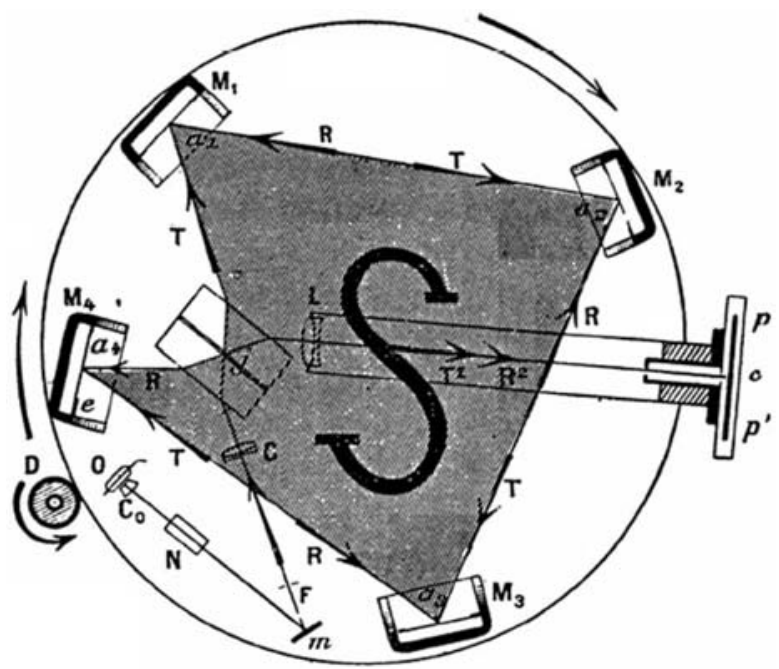


The frequency of light $\omega=k c \equiv c / \hbar$ and (1) allow us to cast $\Delta \varphi_{\mathrm{L}}$ in the more familiar form

$$
\Delta \varphi_{\mathrm{L}}=4 \frac{\Omega S}{\lambda c},
$$

see e.g. Post (1967) and Chow et al. (1985). Here, $\lambda \equiv \lambda /(2 \pi)$ denotes the reduced wave length and $S \equiv \pi r^{2}$ is the area of the circle enclosed by the two counter-propagating beams. It is interesting to note that although (4) was only derived for a circular path it holds true for a closed loop of arbitrary shape.

The expression (3) for the Sagnac phase shift of light shows that $\Delta \varphi_{\mathrm{L}}$ increases quadratically with the time $T$ the light spends in the interferometer. Two methods to increase $T$ offer themselves: (i) to increase the path length by circulating the light many times, and (ii) to use slow light, see Scully and Welch (2004). The first method is utilized in modern optical fiber gyroscopes, which are valuable tools to measure rotation rates to a high precision. Such gyroscopes are sometimes called passive Sagnac interferometers since the light source is located outside of the interferometer. The second approach is based on the fact that in appropriately prepared media the speed of light can be made arbitrarily small. Indeed, light can be (almost) stopped. This phenomenon which has been verified in many spectacular experiments can be used to build a gyroscope, see Zimmer and Fleischhauer (2004, 2006).

Another modern off-spring of Sagnac interferometry is the ring laser gyroscope. In such a device, the lasing medium providing the light source sits inside the interferometer. As a result the clockwise and counter-clockwise propagating waves in this so-called active Sagnac interferometer experience different resonance conditions, giving rise to a beat frequency

$$
\Delta \omega \cong \omega \frac{\delta P}{P}=4 \frac{\Omega S}{\lambda P} .
$$

Here we have made use of (1) and (2). For a detailed discussion of ring laser gyroscopes we refer to the review article by Stedman (1997).

In a seminal experiment, a huge ring laser gyroscope with an area of $16 \mathrm{~m}^{2}$ was used by Stedman and Schreiber et al. (2004), to measure variations in the location of the rotational pole of the Earth to an accuracy of a few centimeters. Moreover, there are strong indications that general relativistic effects connected to gravito-magnetism as discussed in the next section might be detected by these devices, see Scully $(1979,1981)$ and Schleich and Scully (1984). We are confident that future improvements of active as well as passive Sagnac interferometers will open new avenues for tests of general relativity.

\subsection{Atom Interferometer}

Another interesting way to detect rotation consists of replacing in an interferometer light waves by matter waves. The emerging field of matter wave optics is still a young, but rapidly progressing branch of quantum optics, see e.g. Baklanov et al. (1976), Bordé (1989), Berman (1997) and Cronin et al. (2009). It has recently led to sensational discoveries such as laser cooling and atom lasers which were awarded by several Nobel Prizes.

Atomic quantum sensors rely on the wave nature of the center-of-mass motion of an atom, which implies that a single atom moves at the same time along different trajectories, see Rasel et al. (1995). Interference of trajectories is the crucial ingredient of quantum mechanics summarized most beautifully in Young's double-slit experiment and expressed in Feynman's path integral.

During the last decades matter wave optics has turned into an important tool in the ultraprecise monitoring of accelerations and rotations, see Kasevich and Chu (1991), Riehle et 
al. (1991), Gustavson et al. (2000), Gauguet et al. (2006) and Le Gouët et al. (2008). The potential of atom interferometers and atom lasers can be compared with Superconducting Quantum Interference Device (SQUID) sensors, but without the need for cryogenic equipment. Atomic inertial quantum sensors function similarly to atomic clocks, which are today the most-accurate standards for time and frequency measurement. Like atomic clocks, which revolutionized frequency metrology, inertial and rotational sensors using atom interferometers display a high potential for replacing classic state-of-the-art sensors, see Pereira Dos Santos and Landragin (2007) and Lamporesi et al. (2008).

\subsubsection{Enhancement Factor}

The main advantage of using de Broglie rather than light waves in rotation sensors originates from the fact that their wavelength is much shorter, which in principle allows for a much larger Sagnac phase shift. This claim can easily be motivated by the following argument: When we define the mass $m$ of light by the familiar energy relation

$$
m c^{2} \equiv \hbar \omega=\hbar \frac{c}{\lambda}
$$

we can cast the Sagnac phase shift for light given by (4) into the form

$$
\Delta \varphi_{\mathrm{L}}=4 \frac{m}{\hbar} \Omega S .
$$

Moreover, it can be shown, see e.g. Staudemann et al. (1980) and Rauch and Werner (2000), that in complete analogy the corresponding Sagnac phase shift $\Delta \varphi_{M}$ for matter waves after one full circulation around the enclosed area $S$ reads

$$
\Delta \varphi_{\mathrm{M}}=4 \frac{M}{\hbar} \Omega S,
$$

with $M$ being the mass of the massive particle.

Thus, when we compare the phase shift obtained for a light ray, say a helium-neon laser with typical wavelength $\lambda=632.8 \mathrm{~nm}$, to a matter wave of rubidium-87 atoms with mass $M=86.91 \mathrm{u}$, we obtain for their ratio

$$
\frac{\Delta \varphi_{\mathrm{M}}}{\Delta \varphi_{\mathrm{L}}}=\frac{M}{m}=\frac{M c^{2}}{\hbar \omega} \approx 4 \times 10^{10} .
$$

Here, we have assumed that the helium-neon laser and the rubidium atoms enclose the same area $S$. In this case, we gain a factor of $10^{10}$ in resolution taking advantage of matter instead of light waves. However, the problem with matter waves is that one has not yet a source of comparable large particle fluxes as in the case of the laser. Hence, the signal to noise ratio is much worse for matter waves than for light waves, see Scully and Dowling (1993).

\subsubsection{Cold Atoms and the Need for Microgravity}

The fact that the Sagnac phase shift increases quadratically with $T$ is not limited to light waves but also holds true for matter waves. Hence, atoms with an extremely small translational kinetic energy, that is cold atoms, allow us to achieve unusually long transit times $T$. Unfortunately, now we face a problem: Due to the non-vanishing mass atoms are attracted to the Earth. For this reason fountain experiments where the atoms are launched against the 
gravitational field of the Earth and the measurement is performed at the turning point of the motion are of interest. Another method to avoid the grip of gravity on the atoms consists of performing atom interferometer experiments in a microgravity environment as provided for example by a freely falling elevator or satellite, an air plane in parabolic flight or in the International Space Station.

An example for a space version of a quantum test of the equivalence principle is the matter wave explorer of gravity (MWXG) which will employ atomic Mach-Zehnder-type interferometers, see Ertmer et al. (2009). Here, atomic wave packets made out of cold atoms are coherently split, redirect and recombine to observe matter wave interferences, see Rasel et al. (1995). The splitting is achieved by the interaction between the atoms and pulsed light fields generated by two counter-propagating laser beams. An atom absorbs a photon from one of the laser beams and is stimulated by the other laser beam to re-emit another one. In this way twice the recoil of a photon is transferred coherently to the atomic wave (rather than atoms) to generate a new spatial mode of a matter wave, see Müller et al. (2009).

Due to their high symmetry Mach-Zehnder atom interferometers are perfect tools for tests of gravity. For example, the phase shift

$$
\Delta \varphi=\mathbf{k}_{\mathrm{eff}} \cdot \mathbf{a} T^{2}=\mathbf{k}_{\mathrm{eff}} \cdot \mathbf{a} \frac{L^{2}}{v^{2}}
$$

due to an acceleration a is independent of the atomic mass of the particular species. Therefore, this formula implies the equality of the inertial and the gravitational mass.

Moreover, the phase shift $\Delta \varphi$ given by (5) depends only on the square of the drift time $T$ of the atom inside the interferometer and on the momentum difference $\hbar \mathbf{k}_{\text {eff }}$ of the two interfering matter waves. The latter is determined by the photon recoil of the light beam interacting with the atom.

The simplicity of the expression (5) for the phase shift $\Delta \varphi$ suggests the use of atom interferometers as inertial references for measuring absolute accelerations. However, according to (5) the Mach-Zehnder interferometer senses accelerations in only one particular direction defined by the coherently scattered photons.

The sensitivity of the atomic accelerometer increases for a given length scale $L$ of the detection volume and effective photon recoil of the interferometer with the square of the atomic drift time $T$ or the inverse square of the atomic velocity. Thus, for an atomic accelerometer the sensitivity as well as the precision by using ultra-cold atoms in a space-bound experiment would increase by several orders $\left(10^{3}\right.$ to $\left.10^{4}\right)$ of magnitude allowing longer measurement times and providing a more stable environment, see Zoest et al. (2008).

\subsubsection{Present Activities and Goals}

The recent improvement of sensor performance is mainly due to the rapid progress in the development of lasers and in the manipulation of atoms. World wide groups try to develop transportable variants of matter wave gyroscopes, gravimeters and gradiometers and reach for stationary large scale devices and experiments in space, see Nyman et al. (2006), Lamporesi et al. (2008) and De Angelis et al. (2009). Activities aim for both, increasing the sensitivity and, at the same time, improving the sensor's transportability, as these devices should be used in navigation, in Earth observation, metrology and fundamental physics, see Le Gouët et al. (2008). First prototypes of compact one- and three-axes sensors were tested with the gyroscopes and improved variants are under development. They should provide an enhanced short-term sensitivity aiming for a reduction of the integration time by several 
orders of magnitudes, using for example the so-called butterfly configuration. This interferometer was demonstrated recently in a proof-of-principle experiment. Moreover, novel stationary devices operated in large towers are under development in order to investigate their ultimate sensitivity. Another goal is to transfer the knowledge acquired during experiments in extended free fall in drop towers, parabolic flights or in space to improve current ground based precision.

\subsection{Bose-Einstein Condensates}

As mentioned in the preceding subsection the sensitivity of an atom interferometer is determined by the transit time of the atom. Quantum gases at ultralow temperatures permit to extend this time since the atomic cloud expands rather slowly. In this way many novel devices and effects, such as atom interferometers and atomic clocks with unprecedented accuracy, phase transitions or atom lasers have been achieved. In this section we present a historical perspective on the phenomenon of Bose-Einstein condensation and conclude by briefly summarizing recent activities on BEC in microgravity.

\subsubsection{A Brief History}

The unusual state of matter in which all atoms are in the ground state of their center-ofmass motion originates from a paper by Satyendra Nath Bose, in which he derived Planck's radiation law by taking advantage of the statistical properties of light, see Bose (1924). He sent the paper to Einstein and asked for his opinion. Einstein was quite impressed by the work, translated the English manuscript into German and submitted the paper to Zeitschrift für Physik where all articles at that time appeared in German. Einstein also generalized Bose's work in a subsequent paper entitled "Quantentheorie des einatomigen idealen Gases" (Quantum theory of the monoatomic ideal gas), Einstein (1924). At the end of it Einstein concludes:

Die Klammer drückt den Quanteneinfluß auf das Maxwellsche Verteilungsgesetz aus. Man sieht, daß die langsamen Moleküle gegenüber den raschen häufiger sind, als es gemäß Maxwells Gesetz der Fall wäre.

The bracket expresses the influence of the quanta on Maxwell's distribution law. One sees that the slower molecules occur more frequently, as compared to the fast ones, than they would by virtue of Maxwell's law.

In a second paper also published in the Proceedings of the Prussian Academy of Science, Einstein (1925) talks about the possibility of condensation of atoms. Here most of the atoms are in a ground state of a box, very much like the condensation of steam.

Ich behaupte, daß in diesem Falle eine mit der Gesamtdichte stets wachsende Zahl von Molekülen in den 1. Quantenzustand (Zustand ohne kinetische Energie) übergeht, während die übrigen Moleküle sich gemäß dem Parameterwert $\lambda=1$ verteilen. Die Behauptung geht also dahin, daß etwas Ähnliches eintritt wie beim isothermen Komprimieren eines Dampfes über das Sättigungsvolumen. Es tritt eine Scheidung ein; ein Teil »kondensiert«, der Rest bleibt ein »gesättigtes ideales Gas«.

I claim that in this case a number of molecules which always grows with the total density makes a transition to the 1. quantum state (state without kinetic energy), whereas the remaining molecules distribute themselves according to the parameter 
value $\lambda=1$. The claim thus asserts that something similar happens as when isothermally compressing a vapor beyond the volume of saturation. A separation occurs; a part condenses", the rest remains a "saturated ideal gas".

We note that already in 1911 Ladislas Natanson derived in his paper entitled "On the Statistical Theory of Radiation" the Planck radiation formula using the concept of particle indistinguishability, Natanson (1911). According to Spalek (2006) Natanson visited Einstein in Berlin in 1914. However, it is not clear if on this occasion he mentioned his own work to him, since Natanson was a shy person. He became an eminent intellectual after the rebirth of Poland in 1918 and even Rector Magnificus of the Jagiellonian University in Cracow. It is amusing that he also received highest awards in literature for his essays on Shakespeare and the ancient Greek dramas.

\subsubsection{BEC and Microgravity}

It is interesting to speculate if quantum degenerate gases will be of advantage in metrological applications. The ground state of a harmonic trap is a minimum uncertainty state determined by the Heisenberg uncertainty relation between position and momentum. Lowering the trap will adiabatically release the atoms forming an ideal wave packet. The perfect control of the external degrees of freedom is mandatory for minimizing systematic errors such as the Coriolis force on the atoms or wave front curvatures of the light fields acting as beamsplitters for the matter waves. The control of these errors becomes even more critical when we increase the sensitivity of an atom interferometer by extending the transit time. The gain in sensitivity and in accuracy is compensating for the loss in atom number, which only enters as a square root into the sensitivity at the shot noise limit.

In this regard microgravity can be beneficial in several respects. Indeed, it allows us to study quantum gases at temperatures below pico-Kelvins, to obtain de Broglie wavelength of macroscopic dimensions, and to achieve unperturbed evolutions of these distinguished quantum objects for an unusually long duration. Hence, microgravity sets the stage for the physics of ultra-dilute gases and giant matter waves and the control of these macroscopic quantum objects and mixtures in an environment unbiased by gravity. In particular, microgravity is of high relevance for matter waves as it permits the extension the unperturbed free fall of these test particles in a low-noise environment. This is a prerequisite for fundamental tests in the quantum domain such as the equivalence principle or the realization of ideal reference systems.

The QUANTUS team, formed by a consortium of the Leibniz University of Hanover, the Universities of Hamburg, Berlin and Ulm together with ZARM as well as the Max-Planck Institute for Quantum Optics and ENS, realized a compact facility to study a Rubidium $\mathrm{BEC}$ in the extended free fall at the drop tower in Bremen and during parabolic flights. These environments will permit the QUANTUS team to investigate the generation of a BEC and its release from the trap in microgravity, as well as a detailed experimental analysis of decoherence. The remote controlled and miniaturized BEC trap is under test in the drop tower since November 2007.

\section{Gravito-magnetism}

After this overview of seminal experiments dealing with rotation and modern issues related to matter waves, we now turn to the discussion of experimental tests of gravito-magnetism. For this purpose, we first address the notion of rotation in general relativity and motivate 
Table 1 Basic equations of tensor calculus and general relativity including the linearized field equations. The gravitational constant $G$ and the speed of light appear in the definition of the coupling constant $\kappa=$ $(8 \pi G) / c^{4}$. Moreover, $\Lambda$ and $T^{\mu \nu}$ denote the cosmological constant and the contravariant components of the stress-energy tensor, respectively

\begin{tabular}{ll}
\hline Minkowski metric & $\left(\eta_{\mu \nu}\right) \equiv \operatorname{diag}(1,-1,-1,-1)$ \\
Christoffel symbols & $\Gamma^{\mu}{ }_{\alpha \beta} \equiv \frac{1}{2} g^{\mu \nu}\left(g_{\nu \alpha, \beta}+g_{\nu \beta, \alpha}-g_{\alpha \beta, \nu}\right)$ \\
Curvature tensor & $R^{\mu}{ }_{\alpha \beta \gamma} \equiv \Gamma^{\mu}{ }_{\alpha \gamma, \beta}-\Gamma^{\mu}{ }_{\alpha \beta, \gamma}+\Gamma^{\mu}{ }_{\rho \beta} \Gamma^{\rho}{ }_{\alpha \gamma}-\Gamma^{\mu}{ }_{\rho \gamma} \Gamma^{\rho}{ }_{\alpha \beta}$ \\
Ricci tensor and scalar curvature & $R_{\alpha \beta} \equiv R^{\mu}{ }_{\alpha \mu \beta} \quad$ and $\quad R \equiv R_{\mu}^{\mu}$ \\
Einstein's field equations & $R_{\mu \nu}-\frac{1}{2} g_{\mu \nu} R=\kappa T_{\mu \nu}+\Lambda g_{\mu \nu}$ \\
\hline Weak-field approximation & $g_{\mu \nu}=\eta_{\mu \nu}+h_{\mu \nu} \quad$ with $\left|h_{\mu \nu}\right| \ll 1$ \\
Hilbert gauge & $\phi^{\mu \nu}{ }_{, \nu}=0 \quad$ with $\quad \phi_{\mu \nu} \equiv h_{\mu \nu}-\frac{1}{2} \eta_{\mu \nu} h_{\alpha}^{\alpha}$ \\
Linearized field equations & $\square \phi_{\mu \nu}=-2 \kappa T_{\mu \nu}$ \\
\hline
\end{tabular}

the Lense-Thirring metric. We then briefly summarize two recent experiments on gravitomagnetism: (i) the Gravity Probe B experiment (GP-B) and (ii) the laser ranging of the satellites LAGEOS and LAGEOS II. We conclude with an introduction to two-body effects in general relativity and their recent experimental verification by astronomical observations of the double binary pulsar PSR J0737-3039 A/B.

There exist many introductions into the physics of gravito-magnetism. Here we only refer to the text book by Ciufolini and Wheeler (1995) and the articles by Lämmerzahl and Neugebauer (2001) and Mashhoon et al. (2001). The fact that all gravito-magnetic effects give rise to non-geodesic motion and the corresponding relationship between spin-dependent coordinate transformations and spin supplementary conditions is discussed in Barker and O'Connell (1974). Up-to date reviews appear in Ciufolini (2007) and O'Connell (2009a, 2009b).

In order to clarify the sign conventions used throughout this article, we summarize several fundamental equations of tensor calculus and general relativity in Table 1.

\subsection{The Relativistic Notion of Rotation}

In general relativity spacetime is no longer presumed to be flat. Instead, the metric is the basic quantity which incorporates gravity and provides the link between theoretical predictions and experimental results. In the description of experiments in general relativity, one distinguishes between local effects which are determined by the curvature of spacetime in the local neighborhood of an observer, and global effects which involve the coordinate dependence of the metric in an extended region of spacetime. As a result two concepts of rotation exist in general relativity. They are based on the inertial compass and the stellar compass, see Weyl (1924).

Let us first elucidate the term inertial compass. For this purpose we consider a freely falling observer who travels along a timelike geodesic in curved spacetime. He carries with him three spatial coordinate axes (spatial tetrad vectors) which allow him to identify certain directions in space. Moreover, the observer is endowed with three gyroscopes, which are aligned mutually orthogonal with respect to each other and which define the inertial compass. Only when the spatial coordinate axes of the observer do not rotate relative to the three gyroscopes, the observer is said to be freely falling and non-rotating along his world line. In this case, his local reference frame corresponds to a local inertial frame. However, when his spatial coordinate axes rotate relative to the gyroscopes, the observer experiences Coriolis- 
and centrifugal like forces in his local neighborhood: The observer rotates relative to the inertial compass. Hence, the inertial compass provides us with an absolute characterization of the state of motion of the observer's spatial coordinate axes based on a local criterion along his trajectory.

In contrast to this local method to quantify rotation by means of gyroscopes, one could likewise use celestial light sources such as catalog stars as spatial reference frame, see e.g. Soffel (1989). In this way, one takes advantage of the stellar compass, which is defined by the directions of the incident light rays of the "fixed stars" along the world line of the observer. This method represents an alternative way to quantify the rotation of the observer's spatial coordinate axes. Obviously, the tangent vectors of the incident light rays crucially depend on the global aspects of the metric and thus, the stellar compass represents a non-local criterion of rotation in general relativity.

The stellar and inertial compass represent two different ways to determine the rotation of an observer. They give different results for stationary but non-static spacetimes when the "fixed stars" and the observer follow the integral curves of the Killing vector field, see Straumann (2004).

\subsection{Lense-Thirring and Geodetic Precession}

In this subsection, we briefly sketch the so-called Lense-Thirring metric as well as the resulting Lense-Thirring and geodetic precession, see Lense and Thirring (1918) and Thirring (1918) and e.g. Schleich and Scully (1984) and Straumann (2004).

This metric is the starting point for the verification of gravito-magnetism, a notion which most prominently reflects the striking analogy between the linearized field equations of gravitation and electromagnetism, see Table 1. A direct consequence of this analogy is the socalled Lense-Thirring metric whose line element reads in the small-velocity limit

$$
\begin{aligned}
\mathrm{d} s^{2}= & \left(1-\frac{2 G m_{2}}{c^{2} r}\right) c^{2} \mathrm{~d} t^{2}-\left(1+\frac{2 G m_{2}}{c^{2} r}\right)\left(\mathrm{d} x^{2}+\mathrm{d} y^{2}+\mathrm{d} z^{2}\right) \\
& -\frac{4 G S_{z}^{(2)}}{c^{3}} \frac{y}{r^{3}} c \mathrm{~d} t \mathrm{~d} x+\frac{4 G S_{z}^{(2)}}{c^{3}} \frac{x}{r^{3}} c \mathrm{~d} t \mathrm{~d} y .
\end{aligned}
$$

It follows from the special stress-energy tensor of incoherent dust $T^{\mu \nu}=\rho u^{\mu} u^{\nu}$ for a spherically symmetric and homogeneous mass distribution $\rho$ rotating around the $z$-axis with a constant rotation rate. The angular momentum and the mass of the rotating body are denoted by $\mathbf{S}^{(2)}=\left(0,0, S_{z}^{(2)}\right)$ and $m_{2}$, respectively.

The Lense-Thirring precession of a drag-free gyroscope $\left(a^{\mu}=0\right)$ around a massive, spinning body originates from the non-diagonal elements of the Lense-Thirring metric. In this spirit, the Lense-Thirring precession is the general relativistic analogy of the electrodynamic precession of a magnetic needle in an external magnetic field.

On its path $\mathbf{r}(t)$ the angular momentum of the gyroscope $\mathbf{S}^{(1)}$ experiences a precession according to

$$
\frac{\mathrm{d} \mathbf{S}^{(\mathbf{1})}}{\mathrm{d} t}=\boldsymbol{\Omega}^{(1)}(t) \times \mathbf{S}^{(\mathbf{1})}(t),
$$

where the instantaneous precession rate $\boldsymbol{\Omega}^{(1)}(t)$ contains beside the much smaller LenseThirring precession $\boldsymbol{\Omega}_{L T}$ the geodetic precession $\boldsymbol{\Omega}_{G P}$. We note that the latter is also present for a non-rotating body. For the case of the spherical symmetric, massive body the total 
precession rate reads

$$
\boldsymbol{\Omega}^{(1)}(t)=\boldsymbol{\Omega}_{G P}(t)+\boldsymbol{\Omega}_{L T}(t)=\frac{3}{2} \frac{G m_{2}}{c^{2}} \frac{\mathbf{r}(t) \times \mathbf{v}(t)}{r^{3}(t)}+\frac{G}{c^{2}}\left(\frac{3\left(\mathbf{S}^{(2)} \cdot \mathbf{r}(t)\right) \mathbf{r}(t)-r^{2}(t) \mathbf{S}^{(2)}}{r^{5}(t)}\right) .
$$

Here, the vectors $\mathbf{r}$ and $\mathbf{v}$ denote position and velocity of the gyroscope with respect to the center of the massive body.

\subsection{Experiments on Gravito-magnetism}

We now turn briefly to the two most prominent measurements of the Lense-Thirring effect in one-body systems: Gravity Probe B and the LAGEOS satellite experiment, after which we discuss the generalization to two-body systems, especially the double binary system.

\subsubsection{Gravity Probe B}

In order to observe the Lense-Thirring precession, NASA has launched in April 2004 the Gravity Probe B satellite (GP-B), see e.g. Keiser (2009). This satellite travels on a polar orbit around the Earth and carries four mechanical gyroscopes and one telescope. The telescope, which points at the guide star IM Pegasi (HR8703), is used to measure the relativistic precession of the gyroscopes with respect to the "fixed stars". Based on the data, collected between August 2004 and September 2005, the predicted geodetic effect was verified to better than $1 \%$. Unfortunately, due to patch effects on the balls of the gyroscopes the much smaller Lense-Thirring precession has not been confirmed to the expected accuracy so far.

Fortunately, the additional precession of the gyroscopes caused by the patch effects and described by a loxodromic curve can be substracted from the data. As a result a confirmation of the Lense-Thirring effect to $15 \%$ is achieved. However, for a more detailed discussion of all systematic effects we refer to the literature.

According to the Merriam-Websters Collegiate Dictionary (1999) the word loxodrome originates from the Greek words Loxos meaning oblique, and Dromos meaning course. It dates back to ca 1795 and is also referred to as the rhumb line whose definition is:

a line on the surface of the earth that follows a single compass bearing and makes equal oblique angles with all meridians.

When projected onto a plane the loxodrome, that is, the rhumb line becomes a spiral. It is interesting to note that another spiral, namely the Cornu spiral, plays a decisive role in the foundations of quantum mechanics. Indeed, the convergence of the Feyman path integral rests on the Cornu spiral. Hence, as it stands right now, the measurement of the LenseThirring effect due to Gravity Probe B again relies on a spiral.

\subsubsection{LAGEOS Satellites}

Another experiment testing the Lense-Thirring effect is due to Ciufolini and Pavlis (2004), Ciufolini (2007). In contrast to the GP-B mission, here the nodal precession of the two satellites LAGEOS and LAGEOS2 due to the spinning Earth is measured. Each satellite consists of retro-reflectors mounted to a heavy sphere of aluminum-covered brass. The reflectors allow the determination of the Earth-satellite distance with a precision of a few millimeters using laser ranging. This experiment has confirmed the Lense-Thirring effect to an accuracy of approximately $10 \%$. We note that another satellite, called LARES, is currently under construction and will be probably launched in 2010. LARES is designed to increase the accuracy to approximately $1 \%$. 


\subsection{Observation of Gravito-magnetic Effects in a Two-Body System}

So far, we only considered gravito-magnetic effects on test-masses. The purpose of the present subsection is to briefly outline the necessary generalizations encountered for twobody systems.

In the previous subsections, we saw that a single rotating body gives rise to a metric, the so-called Lense-Thirring metric, which incorporates the effect of the rotation of a massive body. For a much smaller mass orbiting around this body we have the conventional one-body system. More generally, in the two-body case, we define the mass and rotation (spin) of the larger (smaller) body by $m_{2}$ and $\mathbf{S}^{(2)}\left(m_{1}\right.$ and $\left.\mathbf{S}^{(1)}\right)$, see Fig. 2.

Turning to the situation where $m_{1}$ and $m_{2}$ are comparable, we know that even in the absence of rotation the problem becomes more difficult so that it was non-trivial to calculate the periastron precession, see Robertson (1938) and Einstein et al. (1938). However, in the weak-field limit, even with inclusion of spin, it turns out that an exact analysis is feasible; see Barker and O'Connell (1975b, 1977). In particular, a Hamiltonian $H$ was obtained which is completely analogous to the corresponding problem in quantum electrodynamics (QED). Thus we find it convenient to refer to use the term "spin" in the generic sense of meaning "rotational angular momentum" in the case of a macroscopic body (and "internal spin" in the case of an elementary particle, which is not of interest in the present context). In fact, $H$ contains spin-orbit and spin-spin coupling terms which compare term by term with the corresponding results for, say, the QED interaction of an $e^{-}$and $\mu^{-}$if we let

$$
e^{2} \longrightarrow(\ldots) G m_{1} m_{2}
$$

where (...) refers to small (but important) coefficients, see O'Connell $(1974,2005)$. In other words, the spin-orbit coupling in the weak-field limit of the two-body system in general relativity (GR) corresponds to the fine structure in QED, whereas the spin-spin coupling in GR corresponds to the hyperfine structure in QED.

The advantage of starting with a Hamiltonian is that it provides analogous paths to the calculation of spin-orbit and spin-spin contributions to both spin precession and orbital precession. With regard to the latter, we find after averaging over a period of the orbit defined by the relative coordinate $\mathbf{r}(t)=\mathbf{r}_{2}(t)-\mathbf{r}_{1}(t)$, that the Kepler ellipse precesses as a whole with angular velocity $\boldsymbol{\Omega}^{*}$ such that

$$
\frac{\mathrm{d} \mathbf{L}}{\mathrm{d} t}=\mathbf{\Omega}^{*} \times \mathbf{L}
$$

Fig. 2 Sketch of a two-body system consisting of two comparable masses $m_{1}$ and $m_{2}$ with center of mass $O$. The orbital angular momentum $\mathbf{L}$ is perpendicular to the orbits 1 and 2, which are defined by the position vectors $\mathbf{r}_{1}(t)$ and $\mathbf{r}_{2}(t)$ of the two-body system. The spin of each mass is denoted by $\mathbf{S}^{(1)}$ and $\mathbf{S}^{(2)}$, respectively

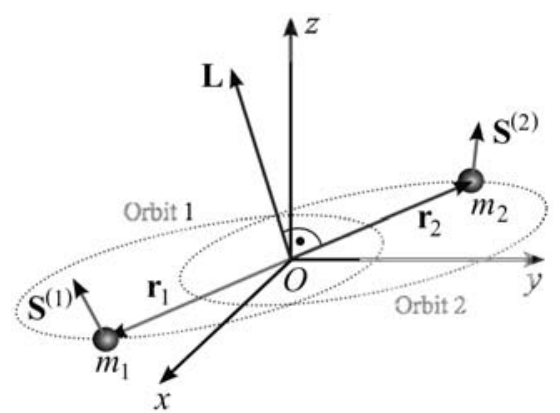


and

$$
\frac{\mathrm{d} \mathbf{A}}{\mathrm{d} t}=\boldsymbol{\Omega}^{*} \times \mathbf{A},
$$

where $\mathbf{L}$ denotes the orbital angular momentum, and $\mathbf{A}$ is the Runge-Lenz vector pointing towards the periastron of the Kepler ellipse. In particular, we note that

$$
\frac{\mathrm{d}}{\mathrm{d} t}(\mathbf{A} \cdot \mathbf{L})=0
$$

as it should, and that $\boldsymbol{\Omega}^{*}$ has contributions from a variety of sources viz.

$$
\boldsymbol{\Omega}^{*}=\boldsymbol{\Omega}^{*(E)}+\boldsymbol{\Omega}^{*(1)}+\boldsymbol{\Omega}^{*(2)}+\boldsymbol{\Omega}^{*(1,2)},
$$

where $\boldsymbol{\Omega}^{*(E)}$ is the Einstein two-body (no spin) contribution, and $\boldsymbol{\Omega}^{*(1)}, \boldsymbol{\Omega}^{*(2)}$ and $\boldsymbol{\Omega}^{*(1,2)}$ refer to the spin 1, spin 2 and spin-spin contribution whose explicit form can be found in Barker and O'Connell (1975b) and O'Connell (2009a). There are also contributions from the quadrupole moments of both bodies, which are important in some cases such as the GPB experiment, see O'Connell (1969), Barker and O'Connell (1975a, 1975b), but we will not consider these here. With regard to spin precession of the individual bodies, we find that

$$
\frac{\mathrm{d} \mathbf{S}^{(1)}}{\mathrm{d} t}=\boldsymbol{\Omega}^{(1)} \times \mathbf{S}^{(1)}
$$

and

$$
\frac{\mathrm{d} \mathbf{S}^{(2)}}{\mathrm{d} t}=\boldsymbol{\Omega}^{(2)} \times \mathbf{S}^{(2)},
$$

where

$$
\boldsymbol{\Omega}^{(1)}=\boldsymbol{\Omega}_{\mathrm{SO}}^{(1)}+\boldsymbol{\Omega}_{\mathrm{SS}}^{(1)}
$$

and similarly for $1 \rightarrow 2$. Here, $\boldsymbol{\Omega}_{\mathrm{SO}}^{(1)}$ and $\boldsymbol{\Omega}_{\mathrm{SS}}^{(1)}$ are the two-body generalizations of the onebody de Sitter geodetic precession and the Lense-Thirring precession, respectively.

It should also be noted that

$$
\frac{\mathrm{d}}{\mathrm{d} t}\left(\mathbf{L}+\mathbf{S}^{(1)}+\mathbf{S}^{(2)}\right) \equiv \frac{\mathrm{d} \mathbf{J}}{\mathrm{d} t}=0 .
$$

In other words, the total angular momentum $\mathbf{J}$ is conserved.

In summary, we are dealing with three main precession rates $\boldsymbol{\Omega}^{*}, \boldsymbol{\Omega}^{(1)}$ and $\boldsymbol{\Omega}^{(2)}$, all of which are derived from three main terms in $H$. Moreover, following Barker and O'Connell (1975b), it is useful to write

$$
\boldsymbol{\Omega}^{*}=\frac{\mathrm{d} \Omega}{\mathrm{d} t} \mathbf{n}_{0}+\frac{\mathrm{d} \omega}{\mathrm{d} t} \mathbf{n}+\frac{\mathrm{d} i}{\mathrm{~d} t} \frac{\mathbf{n}_{0} \times \mathbf{n}}{\left|\mathbf{n}_{0} \times \mathbf{n}\right|},
$$

where $\Omega, \omega$ and $i$ denote the longitude of the ascending node, the argument of the periastron and the inclination of the orbit, respectively, in the reference system of the plane of the sky (the tangent plane to the celestial sphere at the center of mass of the binary system), see Fig. 3. In addition, $\mathbf{n}_{0}$ is a unit vector normal to the plane of the sky directed from the center of mass of the binary system towards the Earth in $z$-direction. The angle between $\mathbf{n}_{0}$ and $\mathbf{n}=\mathbf{L} /|\mathbf{L}|$ is the inclination $i$. In the absence of spin, only the periastron precession is present. 


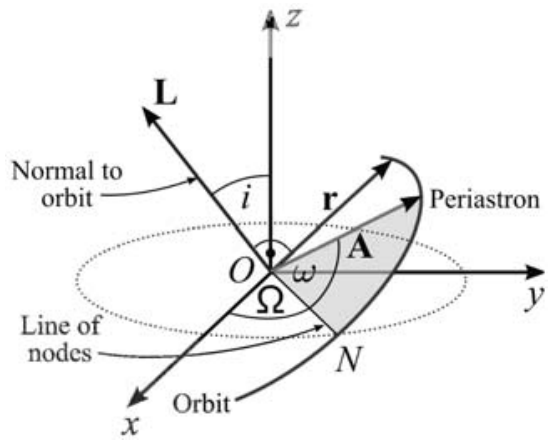

Fig. 3 Illustration of orbital coordinates with the center of mass of the two-body system denoted by $O$. The angles $\Omega, \omega$ and $i$ represent the longitude of the ascending node, the argument of the periastron and the inclination of the orbit in the reference system of the plane of the sky represented by the $x-y$-plane. The relative coordinate of the two-body system $\mathbf{r}(t)=\mathbf{r}_{2}-\mathbf{r}_{1}$ defines the orbit which lies in the plane orthogonal to the orbital angular momentum $\mathbf{L}$. Moreover, the node $N$ is the intersection point of the orbit with the plane of the sky, whereas A stands for the Runge-Lenz vector which points toward the periastron

We turn next to a discussion of specific attempts to measure or observe gravito-magnetic effects. In this context, exact expressions for the various precession angles have been given in the literature, see Barker and O'Connell (1975b) and O'Connell (2009a). As it turns out, the GP-B experiment measures $\boldsymbol{\Omega}^{(1)}$, whereas current astronomical observations attempt to measure both, $\boldsymbol{\Omega}^{(1)}$ and $\boldsymbol{\Omega}^{(2)}$. By contrast, the Ciufolini-Pavlis experiment and the JPL lunar lasing experiment measure $\boldsymbol{\Omega}^{*}$. Moreover, as emphasized by O'Connell (2009a), for a particular system, the magnitude of the periastron and spin-orbit precession depends significantly on the product of the averaged orbital angular velocity

$$
\bar{\omega}=\frac{2 \pi}{T}=\sqrt{\frac{G M}{a^{3}}}
$$

and the gravitational coupling constant

$$
\alpha_{g}=\frac{G M}{c^{2} a},
$$

where $T$ is the period, $a$ is the semi-major axis of the Kepler ellipse and $M=m_{1}+m_{2}$. For illustration we provide some calculated values of $\bar{\omega}$ and $\alpha_{g}$ in Table 2.

In particular, we note that for the double binary pulsar the product $\alpha_{g} \bar{\omega}$ has the largest value $\left(5.6^{\circ} / \mathrm{yr}\right)$; this difference amounting to nearly 4 orders of magnitude, is reflected in the significantly larger precession (both orbital and spin) obtained for the double binary pulsar, compared to Earth related systems. Thus, we are motivated to present more details on the latter system, particularly because the new two-body spin precession effect has already been measured; see Breton et al. (2008). The double binary system, see Burgay et al. (2003), Lyne et al. (2004) and Kramer et al. (2006), is the only system observed so far in which both components are neutron stars which are pulsing. Its distance from the Earth is about $2.2 \times 10^{16}$ miles or $\frac{1}{22}$ times the milky way diameter. It has a semi-major axis of $1.25 R_{\odot}=$ $6.7 \times 10^{5} \mathrm{~km}$ and thus the entire binary could fit within our sun, whose radius we denoted by $R_{\odot}$. It has a very small eccentricity $e=0.088$ and its angle of inclination is $88.69^{\circ}$ so that the system is observed nearly perfectly edge-on. Also it has a very small orbital period of 2.45 hours. Other useful numbers appear in Table 3. 
Table 2 Periastron precession $\frac{\mathrm{d} \omega}{\mathrm{d} t}=\frac{3}{1-e^{2}}\left(\alpha_{g} \bar{\omega}\right)$ and spin-orbit precession $\Omega_{\mathrm{SO}}=\frac{m_{2}+\frac{\mu}{3}}{2 M} \frac{\mathrm{d} \omega}{\mathrm{d} t}$ of $m_{1}$ is calculated for different one-body and two-body systems. The gravitational coupling constant is given by $\alpha_{g}=\frac{G M}{c^{2} a}$, whereas the average orbital angular velocity reads $\bar{\omega}=\frac{2 \pi}{T}=\sqrt{\frac{G M}{a^{3}}}$. Also $M_{\odot}$ is the solar mass, $\mu=\frac{m_{1} m_{2}}{m_{1}+m_{2}}, M=m_{1}+m_{2}$ and $e$ is the eccentricity of the Kepler ellipse. Note the huge values of the periastron precession $\frac{\mathrm{d} \omega}{\mathrm{d} t}$ and the spin-orbit precession $\Omega_{\mathrm{SO}}$ for the Hulse-Taylor pulsar PRS $1913+16$ and the double binary pulsar PSR J0737-3039 A/B

\begin{tabular}{|c|c|c|c|c|c|}
\hline System & $\begin{array}{l}\text { Sun-(Earth } \\
\& \text { Moon) Gyro }\end{array}$ & $\begin{array}{l}\text { Sun- } \\
\text { Mercury }\end{array}$ & $\begin{array}{l}\text { Earth-Gyro } \\
\text { (GP-B) }\end{array}$ & $\begin{array}{l}\text { PSR } \\
1913+16\end{array}$ & $\begin{array}{l}\text { PSR J0737 } \\
-3039 \mathrm{~A} / \mathrm{B}\end{array}$ \\
\hline$a[\mathrm{~km}]$ & $1.5 \times 10^{8}$ & $6 \times 10^{7}$ & $6 \times 10^{3}$ & $2 \times 10^{6}$ & $9 \times 10^{5}$ \\
\hline$M / M_{\odot}$ & 1 & 1 & $3 \times 10^{-6}$ & $\begin{array}{l}1.44 \text { (Pulsar) } \\
1.39 \text { (Com.) }\end{array}$ & $\begin{array}{l}1.34(\mathrm{~A}) \rightarrow m_{2} \\
1.25(\mathrm{~B}) \rightarrow m_{1}\end{array}$ \\
\hline$e$ & 0.017 & 0.206 & 0.0014 & 0.6 & 0.088 \\
\hline$\alpha_{g}$ & $9.8 \times 10^{-9}$ & $3 \times 10^{-8}$ & $7 \times 10^{-10}$ & $2 \times 10^{-6}$ & $4.4 \times 10^{-6}$ \\
\hline $\bar{\omega}\left[\frac{\mathrm{rad}}{\mathrm{s}}\right]$ & $2 \times 10^{-7}$ & $8.3 \times 10^{-7}$ & $10^{-3}$ & $2.2 \times 10^{-4}$ & $7 \times 10^{-4}$ \\
\hline$\alpha_{g} \bar{\omega}\left[\frac{\circ}{\mathrm{yr}}\right]$ & $3.3 \times 10^{-6}$ & $4.5 \times 10^{-5}$ & $1.4 \times 10^{-3}$ & 0.79 & 5.6 \\
\hline$\frac{\mathrm{d} \omega}{\mathrm{d} t}\left[\frac{\circ}{\mathrm{yr}}\right]$ & $10^{-5}$ & $1.2 \times 10^{-4}$ & $3.5 \times 10^{-3}$ & 4.2 & 16.9 \\
\hline$\Omega_{\mathrm{SO}}\left[\frac{0}{\mathrm{yr}}\right]$ & $5.3 \times 10^{-6}$ & $5.8 \times 10^{-5}$ & $1.8 \times 10^{-3}$ & 1.1 & $\begin{array}{l}4.8(\mathrm{~A}) \\
5.1(\mathrm{~B})\end{array}$ \\
\hline
\end{tabular}

Table 3 Some observed properties of the double binary pulsar PSR J0737-3039 A/B

\begin{tabular}{lllllll}
\hline Pulsar & $\begin{array}{l}\text { Mass } \\
{\left[m / M_{\Theta}\right]}\end{array}$ & $\begin{array}{l}\text { Pulse } \\
\text { frequency } \\
{[\mathrm{Hz}]}\end{array}$ & $\begin{array}{l}\text { Pulse } \\
\text { period } \\
{[\mathrm{ms}]}\end{array}$ & $\begin{array}{l}\text { Surface } \\
B \text {-field } \\
{[\mathrm{G}]}\end{array}$ & $\begin{array}{l}\text { Spin-orbit } \\
\text { precession } \\
{\left[{ }^{\circ} / \mathrm{yr}\right]}\end{array}$ & $\begin{array}{l}\text { Time for } 360^{\circ} \\
\text { revolution of spin } \\
{[\mathrm{yr}]}\end{array}$ \\
\hline$A$ & $m_{2}=1.3381$ & 44 & 22.7 & $6 \times 10^{9}$ & & 75 \\
$B$ & $m_{1}=1.2489$ & 0.36 & 2773.5 & $2 \times 10^{12}$ & $4.77 \pm 0.66$ & 71 \\
\hline
\end{tabular}

Its periastron precession has been measured to an enormous accuracy of

$$
\frac{\mathrm{d} \omega}{\mathrm{d} t}=16.899949(68)^{\circ} / \mathrm{yr} \approx 43000 \cdot\left(\frac{\mathrm{d} \omega}{\mathrm{d} t}\right)_{\text {Mercury }} .
$$

This enables $M$ to be determined with great accuracy

$$
M=m_{1}+m_{2}=2.58708(16) \cdot M_{\odot},
$$

where $M_{\odot}$ denotes the solar mass. However, in order to determine the spin-orbit precession, it is necessary to know both $m_{1}$ and $m_{2}$. In particular, $\frac{m_{1}}{m_{2}}$ is obtainable from $a_{1}=\frac{m_{2}}{M} a$ and $a_{2}=\frac{m_{1}}{M} a$ and the measured projected semi-major values $\frac{a_{1} \sin i}{c}$ and $\frac{a_{2} \sin i}{c}$. In addition, $m_{1}$ and $m_{2}$ are obtainable from the Shapiro time delay (delay of $6.2 \mu$ s due to the effect of pulsar $A$ on the light from pulsar $B$ ). As a result, the spin precession of the axis of pulsar $B$ has been observed to be $4.77 \pm 0.66^{\circ} / \mathrm{yr}$, to an accuracy of $13 \%$, in agreement with the twobody spin-orbit calculations of Barker and O'Connell (1975b), as given in the last row of Table 2. Spin-spin contributions are negligible in an astronomical context (because neutron 
stars have very small radii compared to $a$ ). Even for terrestrial systems, they are not as interesting as spin-orbit effects since they are relatively small and do not involve motion. However, they play a role in the conservation of total angular momentum $\mathbf{J}$.

Finally, we note that further observations on the double binary system will undoubtedly lead to improved values for the spin precession.

\section{Gödel's Universe}

So far, we have discussed recent observations of gravito-magnetism manifesting itself in one- and two-body systems. These systems share the property that the metric is asymptotically flat and follows from the weak-field approximation. However, there exist other solutions of Einstein's field equations which exhibit gravito-magnetic effects and are not asymptotically flat. A prominent and illuminative example of such a solution is the so-called Gödel metric which represents a cosmological solution.

In the present section, we briefly introduce Gödel's metric and discuss time travel using a light cone diagram. Moreover, we illustrate the propagation of light in Gödel's Universe by showing a simulation of a particular visualization scenario. We conclude by summarizing an analogy between Maxwell's equations in a curved spacetime and in the vacuum, and Maxwell's equations in flat spacetime but within a gyrotropic medium. This bridge might open the door for a laboratory approximation of Gödel's Universe.

\subsection{Basic Features}

On the occasion of Einstein's 70th birthday, Gödel proposed an exact solution of Einstein's field equations which contains closed timelike world lines, see Gödel (1949a). Due to the peculiar metric expressed by the line element

$$
\mathrm{d} s^{2}=c^{2} \mathrm{~d} t^{2}-\frac{\mathrm{d} r^{2}}{1+\left(\frac{r}{2 a}\right)^{2}}-r^{2}\left(1-\left(\frac{r}{2 a}\right)^{2}\right) \mathrm{d} \phi^{2}-\mathrm{d} z^{2}+2 r^{2} \frac{c}{\sqrt{2} a} \mathrm{~d} t \mathrm{~d} \phi,
$$

it is in principle possible to travel back in time. Here the parameter $a>0$ has the dimension of a length and characterizes the curvature of Gödel's Universe, see e.g. Schücking and Ozsváth (2003) and Hawking and Ellis (2006).

Before we address the startling opportunity of time travel we briefly list some properties of Gödel's metric (7). We start by noting that it is a cosmological solution of Einstein's field equations, see Table 1, with the stress-energy tensor of an ideal fluid

$$
T_{\mu \nu} \equiv\left(\rho+\frac{p}{c^{2}}\right) u_{\mu} u_{\nu}-p g_{\mu \nu}
$$

as source. The line element (7) is expressed in coordinates comoving with the ideal fluid, so that $u^{\mu}=(c, 0,0,0)$. According to the field equations, see Table 1 , the mass density $\rho$ and the pressure $p$ of the ideal fluid are coupled to the cosmological constant $\Lambda$ and to the parameter $a$ by the relations

$$
\kappa\left(\rho+\frac{p}{c^{2}}\right)=\frac{1}{a^{2} c^{2}} \quad \text { and } \quad \kappa p=\Lambda+\frac{1}{2 a^{2}} .
$$

From the characterization of timelike vector fields by Ehlers (1961), one concludes that the volume expansion and the shear tensor vanish for the velocity field $u^{\mu}$ of the ideal fluid 
in Gödel's Universe, see e.g. Hawking and Ellis (2006). In contrast the rotation tensor has non-zero components, which lead to the rotation scalar

$$
\Omega_{\mathrm{G}} \equiv \frac{c}{\sqrt{2} a}>0 .
$$

We note that $\Omega_{\mathrm{G}}$ is inversely proportional to the parameter $a$ and vanishes in the limit $a \rightarrow \infty$ for which (7) reduces to the line element of flat spacetime.

Finally, we emphasize that Gödel's metric allows for five independent Killing vector fields. It is rotational symmetric and its spacetime is homogeneous. Moreover, Gödel's spacetime is stationary but not static which results in gravito-magnetic effects.

In particular, the inertial compass of an observer comoving with the ideal fluid rotates relative to the stellar compass defined by the "fixed stars" of the ideal fluid. This feature stands out most clearly when we consider Gödel's metric for a small rotation scalar $\Omega_{\mathrm{G}} \ll 1$. In this limit we obtain in first order the line element

$$
\mathrm{d} s^{2}=c^{2} \mathrm{~d} t^{2}-\mathrm{d} r^{2}-r^{2} \mathrm{~d} \phi^{2}-\mathrm{d} z^{2}+2 r^{2} \Omega_{\mathrm{G}} \mathrm{d} t \mathrm{~d} \phi
$$

of flat space time in a frame of reference rotating with rate $\Omega_{\mathrm{G}}$. This rotation gives rise to the Sagnac effect of Gödel's Universe, see Delgado et al. (2002) and Kajari et al. (2004).

We conclude by emphasizing that the metric (7) is by no means a realistic model of our universe, since it cannot explain the red-shift observed for the distant stars. Gödel was well aware of this fact and later on published a metric which contains both expansion as well as rotation, see Gödel (1950).

\subsection{Time Travel}

The most puzzling property of Gödel's Universe is the existence of closed timelike world lines. Gödel (1949a) points out in his original article that

$[\ldots]$ it is theoretically possible in these worlds to travel into the past, or otherwise influence the past.

This statement is illustrated most clearly by the light cone diagram of Fig. 4 where quasi "infinitesimal" light cones are attached to several points of spacetime. The light cones are depicted on two planes of constant coordinate time $t$ for three typical radii $r$. On the inner circle, the light cones are only slightly tilted and rather reminiscent of the ones in a rotating reference frame in flat spacetime. However, already the light cones on the next circle with radius $r=2 a$ (critical Gödel radius) are substantially different from the ones in a rotating reference frame in flat spacetime. Whereas in the rotating frame the opening angle of the cones decreases with increasing radius, the light cones in Gödel's Universe are still wide open and touch the plane of constant coordinate time. Hence, the middle circle corresponds to a closed null curve, provided the $z$-component of the curve is constant.

Finally, on the outer radius the light cones have fallen through the plane of constant coordinate time. For this reason the curves

$$
x^{\mu}(\tau)=\left(t_{0}, r_{0}, \alpha \tau, z_{0}\right) \quad \text { with } \quad \alpha=\frac{c}{r_{0} \sqrt{\left(\frac{r_{0}}{2 a}\right)^{2}-1}}
$$

and $2 a<r_{0}$ represent closed timelike world lines. Since it is possible to follow a timelike world line and return to the same spacetime point at a later proper time, Gödel's Universe in principle allows for time travel. 
Fig. 4 Light cone diagram of Gödel's Universe illustrating the possibility of time travel. The $x$ and $y$-coordinates are given by $x=r \cos \phi$ and $y=r \sin \phi$, whereas the trivial $z$-coordinate has been suppressed in this figure. Coordinate time flows vertically

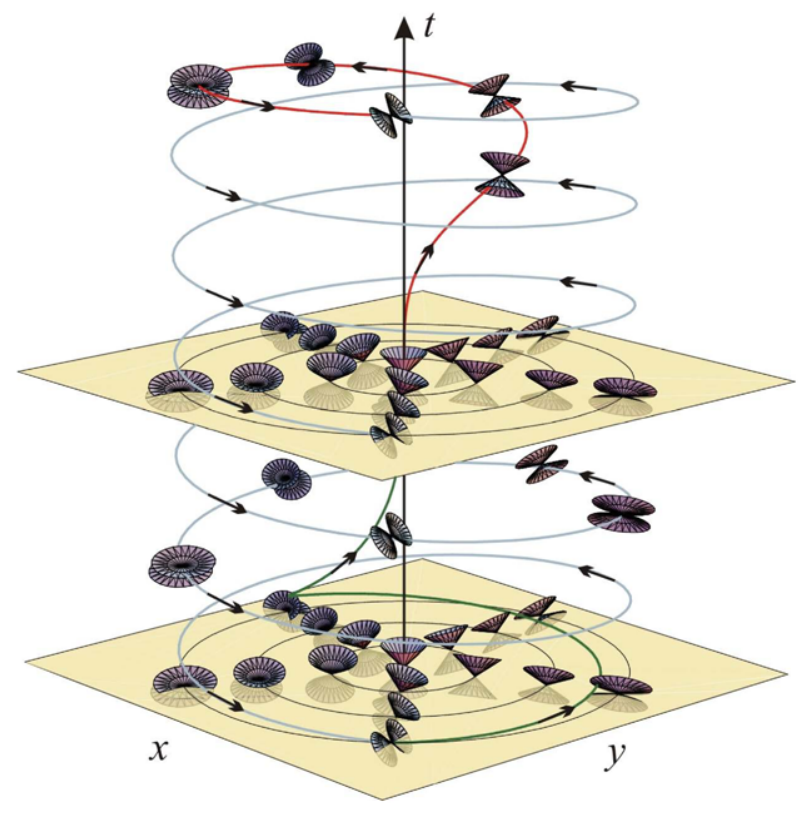

In order to bring out this opportunity of time travel in the most vivid way we start our journey at the origin $r=0$ of the upper plane of constant coordinate time shown in Fig. 4 and seek to return to the very same spacetime point at a later proper time. For this purpose we first follow a timelike world line leading from the origin to a point beyond the critical Gödel radius. The coordinate time along this world line will of course increase during the first part of our journey. After crossing the critical Gödel radius, we take a sharp turn to the "left" in the spacetime diagram Fig. 4 and follow the spiral curve down to earlier coordinate times $t$. Having completed our trip "back into coordinate time", we take another "left" turn onto a world line with increasing coordinate time which brings us back to our initial spacetime point.

In conclusion, at every point of Gödel's Universe there exist closed timelike world lines which pass through it. For more discussion about time travel and Gödel's view on it see Gödel (1949b, 1995).

\subsection{Visualization of Light Propagation}

The rotational structure of Gödel's Universe also manifests itself in the way how an observer visually experiences his surroundings. Due to the non-vanishing rotation tensor light is constantly deflected by Coriolis-like forces and can even return to the point where it was originally emitted. In several articles, see Grave and Buser (2008) and Kajari et al. (2009), we have visualized Gödel's Universe emphasizing effects due to its optical horizon which acts as a mirror and produces multiple images. In the present section we add a novel feature to our visualizations which goes beyond the results presented in Kajari et al. (2009): the appearance of shadows.

Visualizations of relativistic phenomena have become rather popular, see Ertl et al. (1989) and Weiskopf (2000). However, so far shadows have not been considered in this context. Our approach is made possible by exploiting the intrinsic symmetries of the Gödel metric expressed by Killing vector fields, see Grave and Buser (2008). 


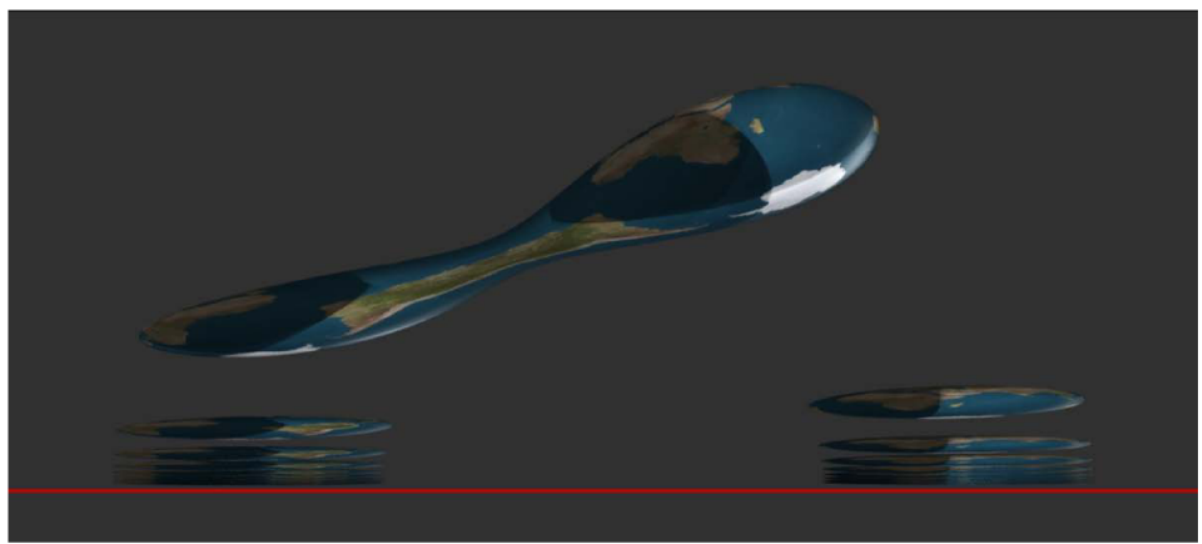

Fig. 5 View on the terrestrial globe by an observer located at the origin of Gödel's Universe. The observer looks in $r$-direction with horizontal and vertical opening angles of $110^{\circ}$ and $90^{\circ}$, respectively. The globe is placed at $r=1.5 a$ and $z=1.2 a$. The multiple images of the globe are a result of distinct paths existing in Gödel's Universe along which light is scattered from the globe to the observer. The whole scenario is illuminated by a point light source coinciding with the observer. Unlike in flat space, in Gödel's Universe shadows are cast on the globe revealing the spots which are illuminated by the light source. The red line marks the $z=0$ plane. We have suppressed the lower half image plane since the globe only appears on the upper one

Kajari et al. (2009) provides a detailed discussion of null geodesics in Gödel's Universe and finds two types of light rays: (i) those which initially propagate towards the observer, and (ii) those which initially propagate away from him. The latter can be redirected to him due to a reflection from the horizon. These two classes of rays create two images of the object, which may even fuse into a single one. Whereas the direct rays display the front of the object, the indirect ones yield the mirror image of its back.

Moreover, Kajari et al. (2009) showed that all null geodesics are of the form of a helix with an axis parallel to the $z$-axis. Since a helix can have an arbitrary number of revolutions there exist a large variety of null geodesics along which light emitted from an object can reach the observer. Each of these paths generates a separate image of an object as exemplified in Fig. 5 by the view on the terrestrial globe. In this scenario the observer is located at the origin of the coordinate system looking in $r$-direction. The globe is positioned at a radial distance $r=1.5 a$ and at the height $z=1.2 a$. All images of the globe are arranged in two different clusters due to the spiral nature of the null geodesics.

In order to obtain a visualization which is more realistic than the ones in Kajari et al. (2009) we have illuminated in Fig. 5 the globe by a light source located at the position of the observer. In flat space no shadows appear in a scenery in which the location of the light source coincides with that of the observer. The paths of the rays from the light source illuminating the scenery and the paths of the rays scattered back to the observer are identical. In contrast, light rays in Gödel's Universe are not invertible due to the intrinsic rotation; light which illuminates the globe takes a different route than light which returns to the observer. This effect leads in Fig. 5 to a superposition of shadows on the surface of the globe.

\subsection{Possible Analogies}

Recently, analogies between light propagation in appropriately prepared media and in curved spacetime have received a lot of attention, see Geoff (2008) and Leonhardt (2009). Indeed, 
three routes to draw these analogies offer themselves: (i) arbitrarily moving media as generalizations of the famous Fizeau experiment, (ii) sound wave propagation in superfluids, and finally (iii) electrodynamics in a gyrotropic medium.

In the present subsection we illuminate the phenomenon of light propagating in a gravitational field from the point of view of light propagating in a gyrotropic medium. Indeed, it was proposed by Tamm (1924) and Skrotskii (1957), that Maxwell's equations in curved spacetime can be formulated in complete analogy to Maxwell's equations in flat spacetime but in the present of a gyrotropic medium, see also Plebanski (1960). We now briefly summarize this formulation, Schleich and Scully (1984).

We start by introducing the spacetime coordinate $x^{\mu} \equiv(c t, x, y, z)$ and the four current $J^{\mu} \equiv\left(c \rho, J_{x}, J_{y}, J_{z}\right)$. In this notation Maxwell's equations for the field tensor $F_{\mu \nu}$ take the relativistic form

$$
\frac{1}{\sqrt{-g}} \frac{\partial}{\partial x^{\nu}}\left(\sqrt{-g} g^{\mu \rho} F_{\rho \beta} g^{\beta \nu}\right)=-\mu_{0} J^{\mu}
$$

and

$$
F_{\alpha \beta, \gamma}+F_{\beta \gamma, \alpha}+F_{\gamma \alpha, \beta}=0
$$

where $g \equiv \operatorname{det} g_{\mu \nu}$.

Next, we identify the components of the field tensor to the three-dimensional fields $\mathbf{E}, \mathbf{D}$, $\mathbf{B}$ and $\mathbf{H}$ via

$$
\begin{aligned}
\frac{1}{c} E_{j} & \equiv F_{0 j}, & D_{j} & \equiv-c \sqrt{-g} F^{0 j}, \\
B_{j} & \equiv-\frac{1}{2} \bar{\varepsilon}_{j l m} F_{l m}, & H_{j} & \equiv-\frac{1}{2} \bar{\varepsilon}_{j l m} \sqrt{-g} F^{l m},
\end{aligned}
$$

with the completely antisymmetric Levi-Civita symbol $\bar{\varepsilon}_{j l m}$. We note that we only use lower indices for $\mathbf{E}, \mathbf{D}, \mathbf{B}$ and $\mathbf{H}$, since there are no co- or contravariant components for these fields according to their definition. However, we still follow the Einstein convention and sum over double indices.

With the identification (12) and the relation $F^{\mu \nu}=g^{\mu \alpha} g^{\nu \beta} F_{\alpha \beta}$, we can establish the constitutive relations

$$
D_{j}=\mu_{j s} E_{s}-c \bar{\varepsilon}_{j l m} g_{l} H_{m}
$$

and

$$
B_{j}=\mu_{j s} H_{s}+\frac{1}{c} \bar{\varepsilon}_{j l m} g_{l} E_{m}
$$

of a gyrotropic medium. Here we have introduced the abbreviations

$$
\mu_{j s} \equiv-\frac{\sqrt{-g}}{g_{00}} g^{j s} \quad \text { and } \quad g_{j} \equiv-\frac{g_{0 j}}{g_{00}} .
$$

Moreover, we find by substitution of the identities (12) into Maxwell's equations (10) and (11) their familiar formulation

$$
\operatorname{div} \mathbf{B}=0, \quad \operatorname{rot} \mathbf{E}=-\frac{\partial \mathbf{B}}{\partial t}
$$


and

$$
\operatorname{div} \mathbf{D}=\frac{\rho}{\epsilon_{0}}, \quad \operatorname{rot} \mathbf{H}=\mu_{0} \mathbf{j}+\frac{1}{c^{2}} \frac{\partial \mathbf{D}}{\partial t},
$$

with the charge density

$$
\rho \equiv \sqrt{-g} \frac{J^{0}}{c}
$$

and the current density

$$
\mathbf{j} \equiv \sqrt{-g} \mathbf{J} .
$$

The electric field vector is like the other vectors defined by $\mathbf{E}=\left(E_{1}, E_{2}, E_{3}\right)$.

So far, we have interpreted the gravitational field as a medium. However, we can also take the opposite point of view and consider the light propagation in a medium as simulating light propagation in a gravitational field. Hence, our strategy for simulating Gödel's metric by a gyrotropic medium is obvious: (i) find from Gödel's line element (7) the metric coefficients $g_{\mu \nu}$, (ii) substitute them into the definitions of $\mu_{j s}$ and $g_{j}$, (15), and (iii) obtain the constitutive relations from (13) and (14).

Obviously this approach can only provide us with the connections between all four fields $\mathbf{E}, \mathbf{B}, \mathbf{D}$ and $\mathbf{H}$. Unfortunately, it is unable to tell us how to implement them in an experiment. The answer to this question goes beyond the scope of the present paper and will be addressed in a future publication. It suffices to say that BECs, meta-materials, see Smith et al. (2004), and electromagnetically induced chirality, see Kästel et al. (2007), represent excellent candidates for such unusual media.

\section{Summary}

In the present article, we have followed the changes in the concept of rotation and its manifestations through several centuries starting from Foucault and Sagnac via Lense-Thirring and Gödel and have finally reached today's rotation sensors based on cold atoms and quantum degenerate gases. In particular, we have discussed several manifestations of gravitomagnetism in the weak-field limit focusing mainly on the relativistic two-body system. In contrast, Gödel's metric serves as an example of a cosmological solution of Einstein's field equations showing gravito-magnetic features. In this context we have pointed out an analogy between electrodynamics in curved spacetime and in a gyrotropic medium, which might open up an avenue for laboratory simulations of light propagation in Gödel's Universe.

During this journey, we have appreciated the fact that atom optics represents a lively field of research at the frontier of experimental general relativity. Moreover, we have emphasized the important role of microgravity in order to achieve long transit times in atom interferometry. This observation serves as one of the many motivations of the QUANTUS project where a BEC is created and observed in a freely falling elevator. Such a system allows us to study the behavior of quantum objects in an environment central to relativity. Indeed, the Gedanken experiment of a freely falling elevator to eliminate locally the effects of gravity is at the very heart of the weak equivalence principle assuming the identity of gravitational and inertial mass.

John Archibald Wheeler frequently told the story that Einstein, during his work on general relativity, had learned about a roofer who had survived his fall off a building. Einstein 
visited him in the hospital to find out what he felt while he was in free fall. The man explained to Einstein that he had experienced no sensation commonly considered as the effect of gravity.

Einstein (1982) himself describes the birth of this corner stone of general relativity as follows:

I was sitting on a chair in my patent office in Bern. Suddenly a thought struck me: If a man falls freely, he would not feel his weight. I was taken aback. This simple thought experiment made a deep impression on me. This led to the theory of gravity.

This paradigmatic change of the concept of gravity stands out most clearly in the poem:

What's the fault of the force on my feet?

What pushes my feet down on the street?

Says Newton, the fault's with the earth's core.

Einstein says, the fault's with the floor;

Remove that and gravity's beat.

(Frances Ruml, 1978, see Wheeler (1983))

The QUANTUS project bears testimony to the fact that atom optics is well on its way to space.

Acknowledgements We thank W. Ertmer, C. Lämmerzahl, H. J. Dittus, I. Ciufolini, J. Ehlers, J. Frauendiener, D. Giulini and H. Pfister for many enlightening discussions, as well as F. Grave, T. Müller, H. Ruder and G. Wunner for the fruitful collaboration. In particular we appreciate conversations with S. Varro, D. Wharam and M. Weidemüller concerning the role of L. Natanson in the creation of the Bose-Einstein statistics. As part of the QUANTUS collaboration, we are grateful for the financial support of the German Space Agency DLR with funds provided by the Federal Ministry of Economics and Technology (BMWi) under grant number DLR 50 WM 0837. We would also like to thank the DFG (German Research Foundation) for the financial support of the project "Visualisierung geschlossener zeitartiger Kurven in der Allgemeinen Relativitätstheorie". The work of R. F. O'C. on this project was carried out in Ulm and he would like to thank the Institute for Quantum Physics for the very gracious hospitality. W. P. S. appreciated the hospitality of the International Space Science Institute (ISSI) during this most exciting workshop in Bern. In particular, he would like to thank R. Treumann and M. Huber for the excellent organization.

\section{References}

A.D. Aczel, Pendulum-Léon Foucault and the Triumph of Science (Washington Square Press, New York, 2003)

Ye.V. Baklanov, B.Ya. Dubetsky, V.P. Chebotayev, Appl. Phys. 9, 171 (1976)

B.M. Barker, R.F. O’Connell, Gen. Relativ. Gravit. 5, 539 (1974)

B.M. Barker, R.F. O'Connell, Phys. Rev. D 11, 711 (1975a)

B.M. Barker, R.F. O'Connell, Phys. Rev. D 12, 329 (1975b)

B.M. Barker, R.F. O'Connell, General relativistic effects in binary systems, in Physics and Astrophysics of Neutron Stars and Black Holes, ed. by R. Giacconi, R. Ruffini. Proceedings of the International School of Physics "Enrico Fermi”, Course LXV (North-Holland, Amsterdam, 1977)

J. Bartlett, J. Kaplan, Bartlett's Familiar Quotations, 17th edn. (Little, Brown and Company, Boston, 2002)

P.R. Berman (ed.), Atom Interferometry (Academic Press, New York, 1997)

Ch.J. Bordé, Phys. Lett. A 140, 10 (1989)

S.N. Bose, Zeitschrift für Physik 26, 178 (1924). Translation: Am. J. Phys. 44, 1057 (1976)

R.P. Breton, V.M. Kaspi, M. Kramer, M.A. McLaughlin, M. Lyutikov, S.M. Ransom, I.H. Stairs, R.D. Ferdman, F. Camilo, A. Possenti, Science 321, 104 (2008)

M. Burgay, N. D’Amico, A. Possenti, R.N. Manchester, A.G. Lyne, B.C. Joshi, M.A. McLaughlin, M. Kramer, J.M. Sarkissian, F. Camilo, V. Kalogera, C. Kim, D.R. Lorimer, Nature 426, 531 (2003) 
W.W. Chow, J. Gea-Banacloche, L.M. Pedrotti, V.E. Sanders, W.P. Schleich, M.O. Scully, Rev. Mod. Phys. 57, 61 (1985)

I. Ciufolini, Nature 449, 41 (2007)

I. Ciufolini, E.C. Pavlis, Nature 431, 958 (2004)

I. Ciufolini, J.A. Wheeler, Gravitation and Inertia (Princeton University Press, Princeton, 1995)

A.D. Cronin, J. Schmiedmayer, D.E. Pritchard, Rev. Mod. Phys. 81, 1051 (2009)

M. De Angelis, A. Bertoldi, L. Cacciapuoti, A. Giorgini, G. Lamporesi, M. Prevedelli, G. Saccorotti, F. Sorrentino, G.M. Tino, Meas. Sci. Technol. 20, 1 (2009)

A. Delgado, W.P. Schleich, G. Süssmann, New J. Phys. 4, 37 (2002)

J. Ehlers, Beiträge zur relativistischen Mechanik kontinuierlicher Medien, in Abhandlungen der Mathematisch-Naturwissenschaftlichen Klasse, vol. 11 (Verlag der Akademie der Wissenschaften und der Literatur, Mainz, 1961)

A. Einstein, How I created the theory of relativity, speech in Kyoto 1922. Phys. Today 35, $45-47$ (1982)

A. Einstein, Sitz. ber. Preuss. Akad. Wiss. 22, 261 (1924)

A. Einstein, Sitz. ber. Preuss. Akad. Wiss. 3 (1925)

A. Einstein, L. Infeld, B. Hoffmann, Ann. Math. 39, 65 (1938)

T. Ertl et al., Visualization in astrophysics, in Eurographics'89: Proceedings of the European Computer Graphics Conference and Exhibition, ed. by W. Hansmann, F.R.A. Hopgood, W. Strasser (Elsevier/North-Holland, Amsterdam, 1989)

W. Ertmer, C. Schubert, T. Wendrich, M. Gilowski, M. Zaiser, T.V. Zoest, E. Rasel, Ch.J. Bordé, A. Clairon, A. Landragin, P. Laurent, P. Lemonde, G. Santarelli et al., Exp. Astron. 23, 611 (2009)

J.B.L. Foucault, Compte-Rendu des séances de l'Académie Des Sci. 32, 135 (1851)

V. Foucault, Recueil des Travaux Scientifiques de Léon Foucault, ed. by C.-M. Gariel (Gauthier, Paris, 1878)

A. Gauguet, B. Canuel, F. Leduc, D. Holleville, N. Dimarcq, A. Clairon, A. Landragin, J. Phys. IV 135, 357 (2006)

B. Geoff, Nature 451, 236 (2008)

K. Gödel, Rev. Mod. Phys. 21, 447 (1949a), reprinted in Gen. Relativ. Gravit. 32, 1409 (2000)

K. Gödel, A remark about the relationship between relativity theory and idealistic philosophy, in Albert Einstein: Philosopher-Scientist, ed. by P.A. Schilpp. The Library of Living Philosophers, vol. VII (Evanston, Illinois, 1949b)

K. Gödel, Proc. Int. Cong. Math. 1, 175 (1950), reprinted in Gen. Relativ. Gravit. 32, 1419 (2000)

K. Gödel, Lecture on rotating universes, in Kurt Gödel: Collected Works, ed. by S. Feferman, J.W. Dawson Jr., W. Goldfarb, C. Parsons, R.N. Solovay. Unpublished Essays and Lectures, vol. III (Oxford University Press, Oxford, 1995)

J.W. Goethe, Faust 1 (1808), English version: Project Gutenberg, translated by G.M. Priest. http://www.einam.com/faust/index.html

J. Le Gouët, T.E. Mehlstäubler, J. Kim, S. Merlet, A. Clairon, A. Landragin, F. Pereira Dos Santos, Appl. Phys. B 92, 133 (2008)

F. Grave, M. Buser, Visiting the Gödel Universe. IEEE Trans. Vis. Comput. Graph. 14, 1563 (2008)

T.L. Gustavson, A. Landragin, M.A. Kasevich, Class. Quantum Gravity 17, 2385 (2000)

S.W. Hawking, G.F.R. Ellis, The Large Scale Structure of Space-Time (Cambridge University Press, Cambridge, 2006)

J. Kästel, M. Fleischhauer, S.F. Yelin, R.L. Walsworth, Phys. Rev. Lett. 99, 073602 (2007)

E. Kajari, R. Walser, W.P. Schleich, A. Delgado, Gen. Relativ. Gravit. 36, 2289 (2004)

E. Kajari, M. Buser, C. Feiler, W.P. Schleich, Rotation in relativity and the propagation of light, in Atom Optics and Space Physics, ed. by E. Arimondo, W. Ertmer, E.M. Rasel, W.P. Schleich. Proceedings of the International School of Physics "Enrico Fermi”, Course CLXVIII (IOS, Amsterdam, 2009)

M. Kasevich, S. Chu, Phys. Rev. Lett. 67, 181 (1991)

G.M. Keiser, Gravity probe B, in Atom Optics and Space Physics, ed. by E. Arimondo, W. Ertmer, E.M. Rasel, W.P. Schleich. Proceedings of the International School of Physics "Enrico Fermi", Course CLXVIII (IOS, Amsterdam, 2009)

M. Kramer, I.H. Stairs, R.N. Manchester, M.A. McLaughlin, A.G. Lyne, R.D. Ferdman, M. Burgay, D.R. Lorimer, A. Possenti, N. D’Amico, J.M. Sarkissian, G.B. Hobbs, J.E. Reynolds, P.C.C. Freire, F. Camilo, Science 314, 97 (2006)

C. Lämmerzahl, G. Neugebauer, The Lense-Thirring effect: from the basic notations to the observed effects, in Gyros, Clocks, Interferometers... . Testing Relativistic Gravity in Space, ed. by C. Lämmerzahl, C.W.F. Everitt, F.W. Hehl (Springer, Berlin, 2001)

G. Lamporesi, A. Bertoldi, L. Cacciapuoti, M. Prevedelli, G.M. Tino, Phys. Rev. Lett. 100, 050801 (2008)

J. Lense, H. Thirring, Phys. Z. 19, 156 (1918). Translation in Gen. Relativ. Gravit. 16, 727 (1984)

U. Leonhardt, Essential Quantum Optics: From Quantum Measurements to Black Holes (Cambridge University Press, Cambridge, 2009) 
A.G. Lyne, M. Burgay, M. Kramer, A. Possenti, R.N. Manchester, F. Camilo, M.A. McLaughlin, D.R. Lorimer, N. D’ Amico, B.C. Joshi, J. Reynolds, P.C.C. Freire, Science 303, 1153 (2004)

B. Mashhoon, F. Gronwald, H.I.M. Lichtenegger, Gravitomagnetism and the Clock effect, in Gyros, Clocks, Interferometers...: Testing Relativistic Gravity in Space, ed. by C. Lämmerzahl, C.W.F. Everitt, F.W. Hehl (Springer, Berlin, 2001)

Merriam-Websters Collegiate Dictionary (Merriam-Webster Incorporated, Springfield, 1999)

T. Müller, M. Gilowski, M. Zaiser, T. Wendrich, W. Ertmer, E.M. Rasel (2009). doi:10.1140/epjd/e200900139-0

L. Natanson, Extraits du Bulletin de l'Academie des Sciences de Cracovie. Série A, 134 (1911). German version: Phys. Z. 12, 659 (1911)

R.A. Nyman, G. Varoquaux, F. Lienhart, D. Chambon, S. Boussen, J.-F. Clément, T. Müller, G. Santarelli, F. Pereira Dos Santos, A. Clairon, A. Bresson, A. Landragin, P. Bouyer, Appl. Phys. B 84, 673 (2006)

R.F. O’Connell, Astrophys. Space Sci. 4, 119 (1969)

R.F. O'Connell, Spin, rotation and C, P and T effects in the gravitational interaction and related experiments, in Experimental Gravitation, ed. by B. Bertotti. Proceedings of the International School of Physics "Enrico Fermi", Course 56 (Academic Press, San Diego, 1974), p. 496

R.F. O’Connell, Class. Quantum Gravity 22, 3815 (2005)

R.F. O'Connell, Gravito-magnetism in one-body and two-body systems: Theory and experiments, in Atom Optics and Space Physics, ed. by E. Arimondo, W. Ertmer, E.M. Rasel, W.P. Schleich. Proceedings of the International School of Physics "Enrico Fermi", Course CLXVIII (IOS, Amsterdam, 2009a)

R.F. O'Connell, Rotation and spin in physics, in Frame-Dragging, Gravitational-Waves and Gravitational Tests, ed. by I. Ciufolini, R. Matzner (Springer, Berlin, 2009b, in press)

F. Pereira Dos Santos, A. Landragin, Phys. World 20(11), 32 (2007)

J. Plebanski, Phys. Rev. 118, 1396 (1960)

E.J. Post, Rev. Mod. Phys. 39, 475 (1967)

E.M. Rasel, M.K. Oberthaler, H. Batelaan, J. Schmiedmayer, A. Zeilinger, Phys. Rev. Lett. 75, 2633 (1995)

H. Rauch, S.A. Werner, Neutron Interferometry: Lessons in Experimental Quantum Mechanics (Oxford University Press, Oxford, 2000)

F. Riehle, T. Kisters, A. Witte, J. Helmcke, C.J. Bordé, Phys. Rev. Lett. 67, 177 (1991)

H.R. Robertson, Ann. Math. 39, 101 (1938)

G. Sagnac, C. R. Acad. Sci., Paris 157, 708 (1913a)

G. Sagnac, C. R. Acad. Sci., Paris 157, 1410 (1913b)

W.P. Schleich, M.O. Scully, General relativity and modern optics, in New Trends in Atomic Physics, Les Houches 1982, Session XXXVIII, ed. by G. Grynberg, R. Stora (North-Holland, Amsterdam, 1984)

K.U. Schreiber, A. Velikoseltsev, M. Rothacher, T. Klugel, G.E. Stedman, D.L. Wiltshire, J. Geophys. Res. 109, B06405 (2004)

E. Schücking, I. Ozsváth, Am. J. Phys. 71, 801 (2003)

M.O. Scully, Suggestion and analysis for a new optical test of general relativity, in Laser Spectroscopy IV, ed. by H. Walther, K.W. Rothe (Springer, Berlin, 1979)

M.O. Scully, J.P. Dowling, Phys. Rev. A 48, 3186 (1993)

M.O. Scully, G.R. Welch, Phys. World 17(10), 31 (2004)

M.O. Scully, M.S. Zubairy, M.P. Haugan, Phys. Rev. A 24, 2009 (1981)

G.B. Skrotskii, Dokl. Akad. Nauk SSSR 114, 73 (1957). Translation: Soviet Phys.-Dokl. 2,226 (1957)

D.R. Smith, J.B. Pendry, M.C.K. Wiltshire, Science 305, 788 (2004)

M.H. Soffel, Relativity in Astrometry, Celestial Mechanics and Geodesy (Springer, Berlin, 1989)

J. Spalek, Letter to Physics Today (2006). http://th-www.if.uj.edu.pl/ztms/jspalek_pl.htm

J.L. Staudemann, S.A. Werner, R. Colella, A.W. Overhauser, Phys. Rev. A 21, 1419 (1980)

G.E. Stedman, Rep. Prog. Phys. 60, 615 (1997)

N. Straumann, General Relativity with Applications to Astrophysics (Springer, Berlin, 2004)

J.E. Tamm, J. Russ. Phys.-Chem. Soc. 56(2-3), 284 (1924)

H. Thirring, Phys. Z. 19, 33 (1918). Erratum: 22, 29 (1921), Translation in Gen. Relativ. Gravit. 16, 712 (1984)

D. Weiskopf, Four-dimensional non-linear ray tracing as a visualization tool for gravitational physics, in Proceedings of the Conference on Visualization 'OO (IEEE Computer Society Press, Los Alamitos, 2000)

H. Weyl, Naturwissenschaften 12, 197 (1924)

J.A. Wheeler, Introduction to general relativity, in Quantum Optics, Experimental Gravitation and Measurement Theory, ed. by P. Meystre, M.O. Scully (Plenum, New York, 1983)

F. Zimmer, M. Fleischhauer, Phys. Rev. Lett. 92, 253201 (2004)

F.E. Zimmer, M. Fleischhauer, Phys. Rev. A 74, 063609 (2006)

T. Zoest, T. Müller, T. Wendrich, M. Gilowski, E.M. Rasel, W. Ertmer, T. Könemann, C. Lämmerzahl, H. Dittus, A. Vogel, K. Bongs, K. Sengstock, W. Lewczko, A. Peters, T. Steinmetz, J. Reichelt, G. Nandi, W.P. Schleich, R. Walser, Int. J. Modern Phys. D 16, 2421 (2008) 